\title{
On the Density of Maximal 1-Planar Graphs
}

\author{
Franz J. Brandenburg ${ }^{1}$, David Eppstein ${ }^{2, \star, \star \star}$, \\ Andreas Gleißner ${ }^{1}$, Michael T. Goodrich ${ }^{2, \star}$, \\ Kathrin Hanauer ${ }^{1}$, and Josef Reislhuber ${ }^{1}$ \\ 1 University of Passau, 94030 Passau, Germany \\ \{brandenb, gleissner, hanauer, reislhuber\}@fim.uni-passau.de \\ 2 Department of Computer Science, University of California, Irvine USA \\ \{eppstein,goodrich\}@ics.uci.edu
}

\begin{abstract}
A graph is 1-planar if it can be drawn in the plane such that each edge is crossed at most once. It is maximal 1-planar if the addition of any edge violates 1-planarity.

Maximal 1-planar graphs have at most $4 n-8$ edges. We show that there are sparse maximal 1-planar graphs with only $\frac{45}{17} n+\mathcal{O}(1)$ edges. With a fixed rotation system there are maximal 1-planar graphs with only $\frac{7}{3} n+\mathcal{O}(1)$ edges. This is sparser than maximal planar graphs. There cannot be maximal 1-planar graphs with less than $\frac{21}{10} n-\mathcal{O}(1)$ edges and less than $\frac{28}{13} n-\mathcal{O}(1)$ edges with a fixed rotation system. Furthermore, we prove that a maximal 1-planar rotation system of a graph uniquely determines its 1-planar embedding.
\end{abstract}

\section{Introduction}

There are numerous results on planar graphs (i.e., graphs that can be drawn without edge crossings), regarding forbidden minors, duality, efficient planarity tests, and straight-line drawings, to name just a few (e.g., see [6, 11, 15]). For instance, it is well-known that planar graphs are sparse, and, in particular, that they have linear density. This is due to the famous Euler Formula, which states that planar graphs have at most $3 n-6$ edges, and this fact has played a critical role in the analysis of the time and area complexities of numerous graph drawing algorithms (e.g., see [5, 10, 18). Moreover, every planar graph can be extended to a maximal planar graph with exactly $3 n-6$ edges by a triangulation of the faces. Maximal planar graphs have a unique embedding on the sphere, which is unique in the plane up to reflection and the choice of the outer face.

Recently, several researchers have investigated graphs that are "almost" planar, where the notion of being "almost" planar is defined to admit crossings in some controlled way and result in linear density. A particular example is 1-planarity, where each edge is allowed to cross at most one other edge. 1-planar graphs were introduced by Ringel [17] in an approach to color a planar graph and its dual.

\footnotetext{
* Supported in part by NSF grant 0830403 , and by the Office of Naval Research MURI grant N00014-08-1-1015.

** Supported in part by NSF grant 1217322 .
}

W. Didimo and M. Patrignani (Eds.): GD 2012, LNCS 7704, pp. 327-338, 2013.

(C) Springer-Verlag Berlin Heidelberg 2013 
There are many properties of 1-planar graphs that are different from their planar counterparts, many of which have yet to be fully explored. For instance, while planar graphs are 4-vertex-colorable, 1-planar graphs are 6-vertex-colorable 4 and 3-colorable for the edges such that black edges are not crossed at all and otherwise a red edge crosses a blue one. In this paper we are interested in the study of properties concerning the density of 1-planar graphs.

\subsection{Prior Work on Properties of 1-Planar Graphs}

A 1-planar graph with $n$ vertices has at most $4 n-8$ edges and this bound is tight. It was first established by Bodendiek et al. 2] and was generalized by Pach and Toth [16. A simple proof is given by Fabrici and Maderas [9].

A 1-planar graph with $4 n-8$ edges is maximally dense. No further edge can be added without violating 1-planarity (in this paper, we consider only graphs that are simple). Bodendiek et al. 3] called these graphs optimal and introduced the extended quadrilateral graphs $X Q_{n}$. An $X Q_{n}$ results from a planar bipartite graph with $n-2$ quadrilateral faces and $2 n-4$ edges by adding a pair of crossing diagonals for each face. Bodendiek et al. showed that there are $X Q_{n}$ 's for $n=8$ and for every $n \geq 10$ and there are no $X Q_{n}$ 's, otherwise.

Schumacher [19] studied 5-connected optimal 1-planar graphs and showed that the $X Q_{n}$ 's have a unique 1-planar embedding on the sphere. The latter result was generalized by Suzuki [20] who dropped the 5-connectivity, and showed that the $X Q_{n}$ 's for $n \geq 10$ have two and the $X Q_{8}$ has eight 1-planar embeddings. Unique 1-planar embeddings were investigated by Korzhik and Mohar [12,13].

1-planar graphs do not always admit straight-line drawings [7]. They are not closed under edge contraction and there are infinitely many minimal non-1planar graphs [13]. The recognition problem of 1-planar graphs is NP-complete 12 , even if the graph is given with a rotation system [1].

Recently, Eades and Liotta showed that maximal RAC graphs are 1-planar [8]. Clearly, every graph has a 1-planar subdivision.

\subsection{Our Results}

A rotation system describes the cyclic ordering of the edges at the vertices as obtained from a drawing. Planarity tests commonly output a rotation system, which is used to compute planar embeddings and straight-line planar drawings in linear time 6, 11, 15]. Here, a rotation system and an embedding can be taken as synonyms, which is almost taken for granted in the graph drawing literature. However, this property no longer holds true for 1-planar graphs. Nevertheless, we show that it holds again for maximal 1-planar graphs.

In this paper, the main focus is on sparse maximal 1-planar graphs. We distinguish maximal 1-planar embeddings and maximal 1-planar graphs. A 1-planar embedding is maximal if no further edge can be added without violating 1planarity. A graph is maximal 1-planar if this holds for all of its 1-planar embeddings. We show that there is a wide range with a number of edges between $\frac{45}{17} n+\mathcal{O}(1)$ and $4 n-8$ for their density. This is surprising. 1-planarity generalizes 
planarity, but maximal 1-planar graphs may be sparser than planar graphs. The sparsity is caused by hermits, which are degree-two vertices enclosed by pairs of crossing edges. Our sparsest 45/17-graphs are constructed from extended quadrilateral graphs $X Q_{8}$ with 11 hermits. In addition, the density of maximal 1-planar embedded graphs decreases to $\frac{7}{3} n+\mathcal{O}(1)$ edges. This is obtained from the combination of fragments with two vertices, four hermits and 14 edges each.

How sparse can we get? We show that the lower bound is at least $2.15 n-\mathcal{O}(1)$ for graphs and at least $2.1 n-\mathcal{O}(1)$ if the rotation system is fixed.

\section{Preliminaries}

We consider simple undirected graphs $G=(V, E)$ with $n$ vertices and $m$ edges. A drawing of a graph is a mapping of $G$ into the plane such that the vertices are mapped to distinct points and each edge is mapped to a Jordan curve between its endpoints. A drawing is plane if the Jordan curve of the edges do not cross and it is 1-plane if each edge is crossed at most once. In 1-plane drawings, crossings of edges with the same endpoint are excluded.

Each plane (1-plane) drawing of a graph implies a rotation system. The rotation at a vertex is the clockwise order of its incident edges or, equivalently, of its adjacent vertices as implied by the drawing. A rotation system of a graph is the list of rotations of all vertices. Note that in general, a given rotation system of a graph may not allow for a plane (1-plane) drawing. Hence, we call a rotation system planar (1-planar) if it admits a plane (1-plane) drawing. A face is a path-connected component of the set of points in the plane not belonging to the drawing. Every point in the boundary of a face belongs to the drawing of a vertex or an edge.

A rotation system of a graph is different from a (topological) embedding. These terms are often identified for planar graphs, since one can be computed from the other in linear time. A (topological) embedding can be obtained from a plane drawing. It specifies the faces by giving a cyclic sequence of the edges which forms its boundary. In a plane drawing every edge occurs in the boundary of at most two faces, and in exactly two faces if $G$ is biconnected. A planar rotation system of a connected graph uniquely determines the embedding on the sphere and vice versa. The same holds for embeddings in the plane, where additionally the outer face has to be defined [14].

Similar to planar embeddings, a 1-planar embedding specifies the faces in a 1-planar drawing. Here, the boundary of a face in a 1-planar embedding is given by a cyclic list of edges and edge segments, where the latter occurs in the case of a crossing. Hence, in 1-planar embeddings, an edge may occur in up to four faces. As with planar graphs, a 1-planar embedding uniquely implies a 1-planar rotation system. Edges or edge segments which are incident to a vertex $v$ and directly succeed in the cyclic boundary list, also directly succeed in the rotation at $v$, and vice versa. However, a 1-planar rotation system does not uniquely determine a 1-planar embedding nor the edges that cross. In fact, deciding whether a rotation system is 1-planar, is NP-hard [1]. 
Let $G$ be a 1-planar embedded graph and denote by $G^{\times}$its planarization. $G^{\times}$ is obtained from $G$ by replacing each pair $e=\{u, v\}$ and $e^{\prime}=\left\{u^{\prime}, v^{\prime}\right\}$ of crossing edges by a new vertex of degree four joined to $u, v, u^{\prime}$, and $v^{\prime}$. Then, $G^{\times}$is a planar embedded graph, where its embedding is inherited from $G$, i. e., the only difference is that edge segments become edges.

A graph is maximal if no further edge can be added without violating its defining property. The maximal planar graphs have exactly $3 n-6$ edges and they are triangulated. The trivial maximal 1-planar graphs are the complete graphs for $n \leq 4$. The $K_{4}$ can be drawn with triangular faces or with faces with two and four vertices.

Lemma 1. In every maximal 1-planar embedding there are at least two and at most four vertices on the boundary of each face.

Proof. Consider the boundary of a face $f$ in a maximal 1-planar embedding. As the boundary of $f$ in the planarization contains at least three edges, the original boundary must contain at least three edges or edge segments. Not all of them can be edge segments since incident edges cannot cross. Vertices incident to the edge are on the boundary of $f$.

Next, suppose that the boundary of the face has more than four vertices. Each pair must be adjacent such that they form a $K_{k}$. This is impossible for $k \geq 5$ [21.

Let us now see how we can construct sparse maximal 1-planar graphs. The basic idea is to have vertices $u$ and $v$ incident to two pairs of crossing edges such that the drawing has a region enclosed by crossing edge segments, which initially contains no other vertex. Thus, the boundary of the region cannot be crossed by any other edge. We place there a vertex $h$ called the hermit, which is adjacent only to $u$ and $v$. This situation is depicted in Fig. 1. A large number of hermits leads to low densities in maximal 1-planar graphs. Note that hermits cannot occur in maximal planar graphs.

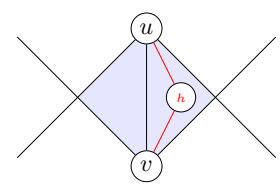

Fig. 1. Hermit $h$ is isolated by two pairs of crossing edges incident to $u$ and $v$.

In a maximal 1-planar embedding, any two vertices in the boundary of a face are connected by an edge as otherwise the edge could be added and drawn in the face without violating 1-planarity. This immediately implies:

Lemma 2. Let $(u, w, v)$ be an uncrossed path (without crossed edges) of a maximal 1-planar embedding of $G$ where $u$ and $v$ are direct successors in the cyclic ordering at $w$. Then $G$ also contains the edge $\{u, v\}$. 
The following observation has been made at several places and holds by a similar argument.

Proposition 1. Let $\{a, b\}$ and $\{c, d\}$ be a pair of crossing edges in a maximal 1-planar embedding of $G$. Then the vertices $a, b, c$, and $d$ form $a K_{4}$.

Lemma 3. If $h$ is a vertex of degree two, its incident edges $\{u, h\}$ and $\{h, v\}$ are uncrossed in any maximal 1-planar embedding of $G$.

Proof. If an edge incident to $h$ is crossed, then $h$ is part of a $K_{4}$ by Proposition 1 . Thus, the degree of $h$ is at least three, a contradiction.

As a consequence, the neighbors $u$ and $v$ of a vertex of degree two are always connected by a supporting edge $\{u, v\}$.

Lemma 4. In a maximal 1-planar embedding of a graph with more than three vertices, every vertex $h$ of degree two is a hermit.

Proof. We show that there are two pairs of crossing edges incident to the vertices $u$ and $v$ adjacent to $h$, which isolate $h . u$ and $v$ are the sole vertices that are located on the boundary of the same face as $h$, because any other vertex that could be joined to $h$ violates maximality. As the path $(u, h, v)$ is uncrossed, it is part of the boundary of exactly two faces $f_{1}$ and $f_{2}$. Both boundaries only consist of edges or edge segments incident to $u$ and $v$. First suppose that $\{u, v\}$ is not part of these boundaries. The boundaries cannot involve any other uncrossed edge since otherwise its endpoints would be part of the boundary, too. Thus, only edge segments remain, which must belong to crossing edges. Hence, $f_{1}$ and $f_{2}$ form an isolated region. Otherwise, $\{u, v\}$ is part of the boundary of, say, $f_{1}$. Let $g$ be the face on the opposite side of $\{u, v\}$. Again, the boundary of $g$ must only consist of edge segments incident to $u$ and $v$, else another vertex could be joined with $h$, crossing $\{u, v\}$. Now, the isolated region consists of $f_{1}, f_{2}$, and $g$.

Lemma 5. In a graph with a maximal 1-planar embedding, every vertex $v$ of degree at least three is covered by $a K_{4}$.

Proof. Consider three consecutive neighbors $a, b$, and $c$ of $v$. If any of the edges $\{v, a\},\{v, b\}$, or $\{v, c\}$ is crossed, then $v$ is part of a $K_{4}$ by Proposition 1]. Otherwise all of the edges $\{a, b\},\{b, c\}$, and $\{c, a\}$ are present by Lemma 2

Note that removing a hermit $h$ does not induce a new hermit $h^{\prime}$. Suppose $h^{\prime}$ is adjacent to $h$ and the removal of $h$ reduces its degree to two. Let $u$ be the other neighbor of $h$. Then it is also adjacent to $h^{\prime}$ by the supporting edge of $h$. Let $v$ be the third neighbor of $h^{\prime}$. If $h^{\prime}$ becomes a hermit, then $\left\{h^{\prime}, v\right\}$ is uncrossed so that initially, $h$ has also been connected to $v$ by Lemma 2 , a contradiction.

The induced subgraph $\hat{G}$ which is obtained from the original graph $G$ by removing all hermits, is called the skeleton of $G$. Likewise, the vertices of $\hat{G}$ are called skeleton vertices. Observe that the removal of a hermit preserves maximal 1-planarity. If it enabled the insertion of a new edge $e$, then $e$ could have been inserted before by crossing one of the hermit's edges, contradicting maximality by Lemma 3 . Thus, we conclude: 
Corollary 1. If the embedding of a graph $G$ is maximal 1-planar, then so is the embedding of its skeleton.

\section{Sparse Maximal 1-Planar Graphs}

In this section we present sparse maximal 1-planar graphs with and without a fixed rotation system. Planar embeddings are uniquely determined by a rotation system. It has been observed by Auer et al. [1] that this does not hold for 1-planar embeddings. We show that it is true again for maximal 1-planar graphs.

Lemma 6. A maximal 1-planar rotation system of a graph $G$ uniquely determines the 1-planar embedding of $G$.

Proof. Korzhik et al. [12] showed that the 1-planar embedding of $G$ is unique if all 1-planar drawings of $G$ have the same pairs of crossing edges and the embedding of their planarization is fixed. First consider a maximal 1-planar embedding of the skeleton $\hat{G}$ of $G$. Any pair of crossed edges in $\hat{G}$ is covered by a $K_{4}$ by Proposition 1 The embeddings of the $K_{4}$ s are determined by the rotation system [1]. Thus, the fixed rotation system of $\hat{G}$ implies the same pairs of crossing edges in any embedding of $\hat{G}$. This is also true for $G$ as the edges incident to hermits cannot cross by Lemma 3 . The embedding of the planarization is also determined by the rotation system.

\subsection{Graphs with Rotation Systems}

First we construct a graph $G=(V, E)$ along with its rotation system. Let $t>5$ be integer. The instance for $t=8$ can be seen in Fig. 2, Let the set of vertices be

$$
V=\bigcup_{i=1}^{t}\left\{a_{i}, b_{i}\right\} \cup \bigcup_{i=2}^{t-1}\left\{c_{i}\right\} \cup \bigcup_{i=1}^{t-1}\left\{d_{i}\right\} \cup \bigcup_{i=2}^{t}\left\{e_{i}\right\} \cup \bigcup_{i=2}^{t-2}\left\{f_{i}\right\}
$$

where $a_{i}$ and $b_{i}$ are skeleton vertices and $c_{i}, d_{i}, e_{i}$, and $f_{i}$ are hermits.

The rotation system is defined by the following cyclic adjacencies. For simplicity, we skip the special cases $i \leq 1$ and $i \geq t-1$. Thus, ignore the entries referring to non-existent vertices. For even $i$ with $1 \leq i \leq t$ define

$$
\begin{array}{lr}
a_{i}: & \left(b_{i+1} d_{i} a_{i+1} c_{i} a_{i-1} e_{i-1} b_{i-1} a_{i-2} f_{i-1} b_{i} f_{i} a_{i+2}\right), \\
b_{i}: & \left(a_{i+1} a_{i} a_{i-1} d_{i-1} b_{i-1} c_{i} b_{i+1} e_{i}\right), \\
c_{i}:\left(a_{i} b_{i}\right), d_{i}: & \left(a_{i} b_{i+1}\right), e_{i}:\left(b_{i} a_{i+1}\right), \text { and } f_{i}:\left(a_{i}, a_{i+1}\right) .
\end{array}
$$

The adjacencies for odd $i$ are the same except that the cyclic order is reversed.

By Lemma 6 we can testify that $G$ is maximal 1-planar by observing a single embedding.

$G$ contains $n=2 t+3(t-2)+(t-3)=6 t-9$ vertices. The sum of degrees is

$$
\underbrace{5+9+12(t-4)+9+5}_{a \text {-vertices }}+\underbrace{3+8(t-2)+3}_{b \text {-vertices }}+\underbrace{2(3(t-2)+t-3)}_{\text {hermits }}=28 t-48 \text {. }
$$




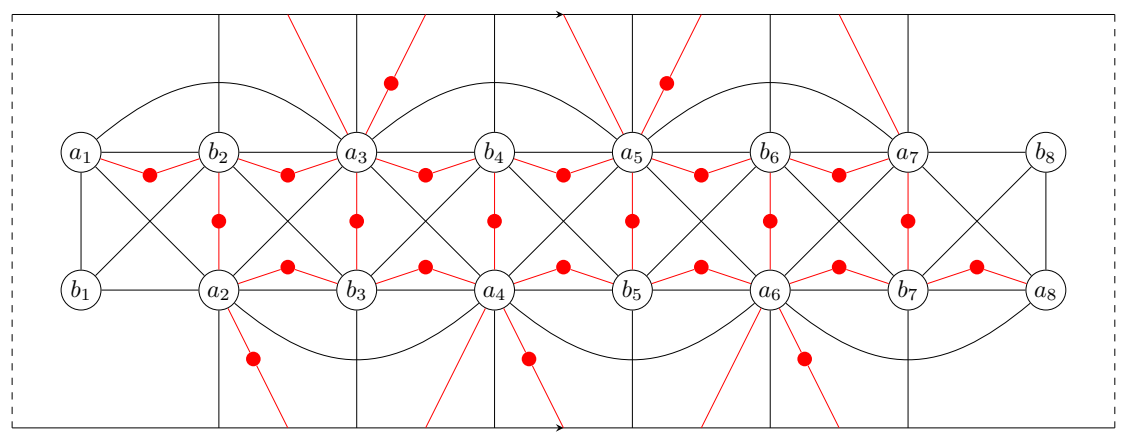

Fig. 2. Graph with $m=\frac{7}{3} n-3$, which is maximal 1-planar for the given rotation system. The top and bottom side of the drawing is identified yielding the surface of a cylinder, which is equivalent to the plane.

Thus, $G$ has $m=14 t-24=\frac{7}{3} n-3$ edges. This result improves the earlier $\frac{5}{2}$ bound from [1].

Theorem 1. There are arbitrarily large graphs with $m=\frac{7}{3} n-3$ edges, which are maximal 1-planar for a fixed rotation system.

\subsection{Graphs without Rotation Systems}

Now we construct sparse maximal 1-planar graphs with density $\frac{45}{17}$. The $45 / 17-$ graphs consists of copies of the extended quadrilateral graphs $X Q_{8}$, where two vertices and an edge are shared by all copies. Each $X Q_{8}$ hosts 11 hermits, and thus adds 17 own vertices and 45 edges, see Fig. 3(a).

$X Q_{8}$ is the only maximal 1-planar graph with maximum density $4 n-8$ [3], but it has different 1-planar drawings, which all end up in the same picture but with different rotation systems and the vertices at different positions.

Theorem 2. There are arbitrarily large, maximal 1-planar graphs with $m=$ $\frac{45}{17} n-\frac{84}{17}$ edges.

Proof. The graphs consist of $t$ copies of the $X Q_{8}$ graph, which is filled with hermits, as shown in Fig. 3(a). Two vertices $a$ and $b$ (in blue) of each copy and the edge $(a, b)$ are identified. Finally add another late hermit, see Fig. $3(\mathrm{~b})$ for an example of the graph with $t=4$.

Obviously, this graph is 1-planar. It is also maximal 1-planar since the given 1-planar drawing is unique up to the permutation of the components and to the placement of each hermit in the faces next to its supporting edge.

According to Suzuki [21], $X Q_{8}$ has eight 1-planar embeddings with different rotation systems. However, when the hermits are added and the vertices $a$ and $b$ are fixed, only one 1-planar drawing remains.

Each hermit $h$ is completely enclosed by a pair of crossing edges from its neighbors $u, v$ to both sides of the edge $(u, v)$. Hence, $h$ can be placed to either side of this edge, and $h$ cannot be connected to any other vertex. 
(a)

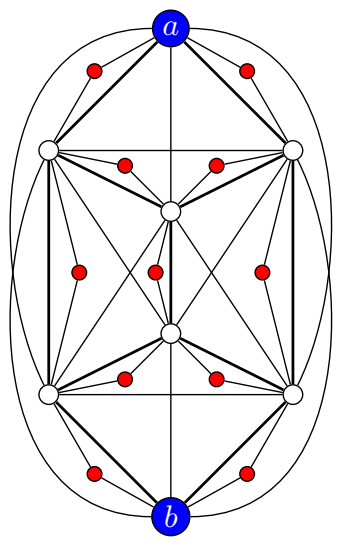

(b)

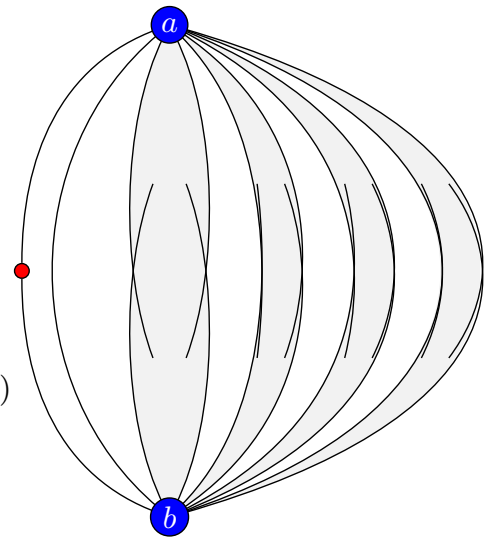

Fig. 3. (a) $X Q_{8}$ graph filled with hermits. The edge $\{a, b\}$ is not shown. (b) Example of the $45 / 17$-graph with $m=\frac{45}{17} n-\frac{84}{17}$, which is composed of $t=4$ filled $X Q_{8}$ s. The $a$-vertices and the $b$-vertices are identified each.

The graph is biconnected with the poles $a$ and $b$. Its components can be permuted arbitrarily, but all drawings are equivalent up to the automorphism resulting from the permutation. All vertices from the copies of the $X Q_{8}$ but the two poles are fully enclosed by crossing edges. Hence, no further edge can be added without violating 1-planarity.

For integers $n$ other than $n=17 t+3$ for some $t \geq 1$ we can replace the late hermit by a small 1-planar graph with at most 16 vertices, and so obtain a sparse maximal 1-planar graph of size $n$.

Corollary 2. For every $n$ there are maximal 1-planar graphs with at most $\frac{45}{17} n+$ $\mathcal{O}(1)$ many edges.

Clearly, there are maximal 1-planar graphs of density $r=\frac{p}{q}$ with integers $p, q$ with $2.64 \leq r \leq 4$, which can be obtained from graphs as in Fig. 3(b) by removing some hermits.

\section{Lower Bounds}

In this section, we will establish lower bounds on the density of maximal 1-planar graphs, both for fixed and free rotation systems.

Let $G$ be a graph with a maximal 1-planar embedding and $\hat{G}$ its skeleton. Let $\hat{n}$ and $\hat{m}$ be the number of vertices and edges in $\hat{G}$. We start with an observation:

Lemma 7. Every edge $\{u, v\}$ of $\hat{G}$ is covered by a $K_{4}$.

Proof. By Proposition[1]we only have to deal with the case that $\{u, v\}$ is uncrossed. Let $f_{1}$ and $f_{2}$ be the faces bordering $\{u, v\}$. Each of the faces must be bounded 
by at least three edges or edge segments, from which one is $\{u, v\}$. If at least one of $f_{1}$ and $f_{2}$ has no further vertex on its boundary, then it is bounded by two edge segments belonging to a pair of crossing edges incident to $u$ and $v$. Hence, by Proposition 1 there is a $K_{4}$ containing $\{u, v\}$. Otherwise, as $f_{1}$ and $f_{2}$ cannot be bounded by the same set of edges or edge segments, let $f_{1}$ and $f_{2}$ have the distinct vertices $w_{1}$ and $w_{2}$ on their boundaries, respectively. $w_{1}$ and $w_{2}$ must be adjacent as otherwise, it would be possible to join them by the edge $\left\{w_{1}, w_{2}\right\}$ crossing $\{u, v\}$. Additionally, $w_{1}$ and $w_{2}$ are each adjacent to both $u$ and $v$ by sharing the boundaries of $f_{1}$ or $f_{2}$. Thus, $u, v, w_{1}$, and $w_{2}$ form a $K_{4}$.

Definition $1\left(K_{4}\right.$ network $\left.\mathcal{K}_{4}(G)\right)$

The $K_{4}$ network $\mathcal{K}_{4}(G)=(\mathcal{V}, \mathcal{E}, \omega: \mathcal{E} \rightarrow\{s, w, m\})$ of $G$ is a simple, weighted graph whose vertex set $\mathcal{V}$ consists of all $K_{4}$ s in $\hat{G}$.

$A$ pair of $K_{4} s$ is adjacent if it shares a vertex in $G$. The weight of an edge $e$ is determined by the number of common vertices of the $K_{4} s$ it connects. We say that $e$ is a strong link with $\omega(e)=s$ if the $K_{4}$ s share three vertices, a weak link with $\omega(e)=w$ if they share two vertices, and a micro link with $\omega(e)=m$, otherwise.

Note that two distinct $K_{4} \mathrm{~s}$ cannot share all four vertices if $G$ is simple. By Lemma 7, every edge is covered by a $K_{4}$. Since $G$ is connected, so is $\hat{G}$ and we can immediately derive from Lemma 7

Corollary 3. The $K_{4}$ network $\mathcal{K}(G)$ is connected.

Consider again the skeleton $\hat{G}$ of $G$. Using the $K_{4}$ network as an auxiliary structure, we are able to establish bounds on the density of $\hat{G}$.

Lemma 8. The skeleton $\hat{G}$ of $G$ has at least $\frac{7}{3} \hat{n}-\frac{10}{3}$ edges.

Proof. Compute a minimum spanning tree of $\mathcal{K}_{4}(G)$ with the algorithm by Jarník and Prim. The preference ordering on the edges is $s<w<m$, i. e., whenever possible, enlarge the tree with strong links, then weak links. If a micro link has to be used and there are multiple candidate $K_{4}$ s that all have the same vertex $u$ of an already visited $K_{4}$ in common, choose one that contains a vertex adjacent to $u$ which is an immediate successor of an already visited vertex $v$ in the rotation system of $u$. Note that such a $K_{4}$ always exists, see the proof of Lemma 7 . We will later refer to $v$ as the predecessor of this $K_{4}$ at $u$.

Concurrently, construct a kind of $K_{4}$-spanning tree on $\hat{G}$. Recall that every vertex is covered by a $K_{4}$. We utilize this fact in order to uniquely assign a set of edges to each vertex.

Consider an iteration of the algorithm in which a $K_{4} k$ is connected to the previous spanning tree by a link $l$. If all vertices of $k$ are part of some $K_{4}$ that has already been visited by the spanning tree algorithm, there is nothing to do. See Fig. 4 for an illustration of the remaining cases. If $l$ is a strong link, the iteration step adds exactly one vertex $v$ along with the three incident edges that are part of $k$ to the $K_{4}$-spanning tree of $\hat{G}$. Assign these three edges to $v$. If $l$ is a weak link, the iteration step adds two vertices of $k$ and five edges, so $\frac{5}{2}$ edges can be assigned to each of both vertices. In the case that one of the two vertices of $k$ has 

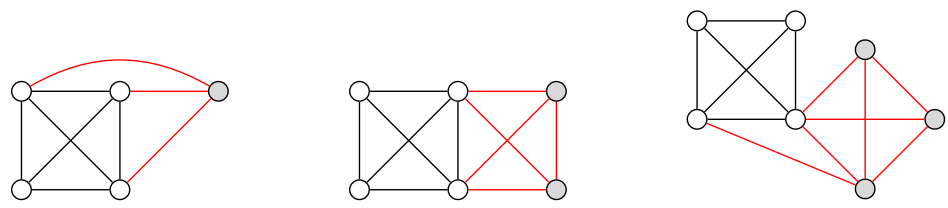

Fig. 4. Edge assignments at strong, weak, and micro links

already been visited, the remaining vertex is added along with its three incident edges. Finally, $l$ might by a micro link. In this case, three vertices of $k$ are added and all six edges. Furthermore, there is a vertex $u$ in $k$ that is also part of an already visited $K_{4}$ and induced the micro link. Now consider the predecessor $v$ of $k$ at $u$. If $\{u, v\}$ is uncrossed, then $v$ and one of the vertices of $k$ reside on the boundary of the same face so they are connected. Otherwise, consider the edge crossing $\{u, v\}$. Again, one of the vertices of $k$ is on the boundary of the same face as one of this edge's endpoints and, therefore, adjacent. Thus, seven edges are assigned to three vertices.

By Corollary 3. the spanning tree algorithm reaches all vertices. This way, we assigned all vertices except for those of the initial $K_{4}$ at least $\frac{7}{3}$ edges. Consider the $K_{4}$ the algorithm starts at. Transferred to the $K_{4}$-spanning tree on $\hat{G}$, this adds four vertices and only six edges, which is a constant error of $\frac{10}{3}$.

The assignment is unique, since an edge is only considered for assignment if one end point has already been visited and the other one is incident to a vertex that is visited at this particular moment. So in total, $\hat{G}$ has at least $\frac{7}{3} \hat{n}-\frac{10}{3}$ edges.

Next, we establish an upper bound on the number of hermits a skeleton can support.

Recall that every hermit needs to be trapped between exactly two crossings. Furthermore, two crossing edges can shield at most four hermits from one another. Denote by $\hat{E}_{c} \subseteq \hat{E}$ the set of crossed edges in the skeleton and $\hat{E}_{p}=\hat{E} \backslash \hat{E}_{c}$. Let $H$ contain all hermits of $G$ and let $C$ be the set of crossings in $G$, which are the same as in $\hat{G}$. From the above argument, we derive

$$
2\left|\hat{E}_{c}\right|=4|C| \geq 2|H| \Rightarrow|H| \leq\left|\hat{E}_{c}\right| \text {. }
$$

Assume now that $G$ 's rotation system is free. Consider the supporting edge $\{u, v\}$ of a hermit $h$. It can always be routed without crossings alongside the path $(u, h, v)$. Furthermore, any uncrossed edge can support at most one hermit, otherwise, there is an embedding such that two or more hermits are not shielded from each other by a crossing. This would allow for the insertion of an edge between them, thus contradicting the maximality of $G$. Subsequently, we also have

$$
|H| \leq\left|\hat{E}_{p}\right| \text {. }
$$

If we combine Inequations 1 and 2 and express the fraction of crossed edges by a variable $\lambda$, we obtain

$$
\begin{aligned}
& a \leq \lambda \text { and } \\
& a \leq(1-\lambda),
\end{aligned}
$$


where $a$ is the ratio of edges in $\hat{G}$ that can be augmented by a hermit. With $0 \leq \lambda \leq 1$, we can bound the maximum value of $a$.

Lemma 9. The ratio of edges that can be augmented by a hermit in a free rotation system is at most $\frac{1}{2}$.

Denote by $b$ the skeleton density, i. e., $\hat{m}=b \cdot \hat{n}$. Each hermit adds exactly one vertex and two edges, so we can express the number of vertices and edges in $G$ as follows:

$$
\begin{array}{r}
n=\hat{n}+a \cdot \hat{m}=\hat{n}+a \cdot b \cdot \hat{n}=\hat{n}(1+a \cdot b) \\
m=\hat{m}+2 a \cdot \hat{m}=b \cdot \hat{n}+2 a \cdot b \cdot \hat{n}=\hat{n}(b+2 a \cdot b)
\end{array}
$$

This leads us directly to the density of $G$, which is

$$
\frac{m}{n}=\frac{b+2 a b}{1+a b} .
$$

With this at hand, we establish the following theorem:

Theorem 3. A maximal 1-planar graph $G$ with a free rotation system has at least $\frac{28}{13} n-\frac{10}{3} \approx 2.15 n$ edges.

Proof. Express by $\rho(a, b)=\frac{b+2 a b}{1+a b}$ the density of $G$ in dependence of $a$ and $b$. With Lemmata 8 and 9, we are able to constrain $a$ and $b$. To simplify calculations, we first argue as if the skeleton had $\frac{7}{3} \hat{n}$ instead of $\frac{7}{3} \hat{n}-\frac{10}{3}$ edges. Thus, we have $0 \leq a \leq \frac{1}{2}$ and $b \geq \frac{7}{3}$. Then, inside this two-dimensional space, $\rho$ decreases with $a$ going from 0 to $\frac{1}{2}$, while it increases with $b$. Hence, $\rho$ takes its minimum value within the definition space at $a=\frac{1}{2}$ and $b=\frac{7}{3}$, which is $\rho\left(\frac{1}{2}, \frac{7}{3}\right)=\frac{28}{13}$. If we subtract $\frac{10}{3}$ again from the final term, we effectively only overestimate the number of hermits, which reduces the density even further. Therefore, we obtain that $\frac{28}{13} n-\frac{10}{3}$ is a lower bound on the number of edges of $G$.

However, if $G$ is provided with a fixed rotation system, then an edge may support arbitrarily many hermits. For example, if the rotation system of the graph depicted in Fig. 3(b) was not free, then a hermit could be inserted between each subsequent pair of $X Q_{8}$ subgraphs, but there is only one supporting edge $\{a, b\}$. As a consequence, Inequation 2 no longer holds here and we have $0 \leq a \leq 1$. Nevertheless, we can use the same argument to establish a lower bound on the density of graphs with fixed rotation systems as we did in the proof of Theorem 3. Setting $a=1$, we obtain:

Theorem 4. A maximal 1-planar graph $G$ with a prescribed rotation system has at least $\frac{21}{10} n-\frac{10}{3} \approx 2.1 n$ edges.

\section{Conclusion}

We have shown that maximal 1-planar graphs can be sparser than maximal planar graphs and that the rotation system uniquely determines the embedding.

Is maximal 1-planarity NP-hard? 


\section{References}

1. Auer, C., Brandenburg, F.J., Gleißner, A., Reislhuber, J.: On 1-Planar Graphs with Rotation Systems. Tech. Rep. MIP-1207, Fakultät für Informatik und Mathematik, Universität Passau (2012)

2. Bodendiek, R., Schumacher, H., Wagner, K.: Bemerkungen zu einem Sechsfarbenproblem von G. Ringel. Abhandlungen aus dem Mathematischen Seminar der Universität Hamburg 53, 41-52 (1983)

3. Bodendiek, R., Schumacher, H., Wagner, K.: Über 1-optimale Graphen. Mathematische Nachrichten 117, 323-339 (1984)

4. Borodin, O.V.: A New Proof of the 6 Color Theorem. Journal of Graph Theory 19(4), 507-521 (1995)

5. De Fraysseix, H., Pach, J., Pollack, R.: How to draw a planar graph on a grid. Combinatorica 10(1), 41-51 (1990)

6. Di Battista, G., Eades, P., Tamassia, R., Tollis, I.G.: Graph Drawing: Algorithms for the Visualization of Graphs. Prentice-Hall (1999)

7. Hong, S.-H., Eades, P., Liotta, G., Poon, S.-H.: Fáry's Theorem for 1-Planar Graphs. In: Gudmundsson, J., Mestre, J., Viglas, T. (eds.) COCOON 2012. LNCS, vol. 7434, pp. 335-346. Springer, Heidelberg (2012)

8. Eades, P., Liotta, G.: Right Angle Crossing Graphs and 1-Planarity. In: van Kreveld, M., Speckmann, B. (eds.) GD 2011. LNCS, vol. 7034, pp. 148-153. Springer, Heidelberg (2012)

9. Fabrici, I., Madaras, T.: The Structure of 1-Planar Graphs. Discrete Mathematics 307(7-8), 854-865 (2007)

10. Goodrich, M.T., Wagner, C.G.: A Framework for Drawing Planar Graphs with Curves and Polylines. In: Whitesides, S.H. (ed.) GD 1998. LNCS, vol. 1547, pp. 153-166. Springer, Heidelberg (1999)

11. Kaufmann, M., Wagner, D. (eds.): Drawing Graphs. LNCS, vol. 2025. Springer, Heidelberg (2001)

12. Korzhik, V.P., Mohar, B.: Minimal Obstructions for 1-Immersions and Hardness of 1-Planarity Testing. In: Tollis, I.G., Patrignani, M. (eds.) GD 2008. LNCS, vol. 5417, pp. 302-312. Springer, Heidelberg (2009)

13. Korzhik, V.P.: Minimal Non-1-Planar Graphs. Discrete Math. 308(7), 1319-1327 (2008)

14. Mehlhorn, K., Mutzel, P.: On the Embedding Phase of the Hopcroft and Tarjan Planarity Testing Algorithm. Algorithmica 16, 233-242 (1995)

15. Nishizeki, T., Rahman, S.: Planar Graph Drawing. World Scientific (2004)

16. Pach, J., Tóth, G.: Graphs Drawn with Few Crossings per Edge. Combinatorica 17, 427-439 (1997)

17. Ringel, G.: Ein Sechsfarbenproblem auf der Kugel. Abhandlungen aus dem Mathematischen Seminar der Universität Hamburg 29, 107-117 (1965)

18. Schnyder, W.: Embedding Planar Graphs on the Grid. In: Proc. SODA, pp. 138148 (1990)

19. Schumacher, H.: Zur Struktur 1-planarer Graphen. Mathematische Nachrichten 125, 291-300 (1986)

20. Suzuki, Y.: Optimal 1-planar Graphs which Triangulate other Surfaces. Discrete Mathematics 310(1), 6-11 (2010)

21. Suzuki, Y.: Re-embeddings of Maximum 1-Planar Graphs. SIAM J. Discrete Math. 24(4), 1527-1540 (2010) 